\title{
Fractional Stochastic Differential Equations with Hilfer Fractional Derivative: Poisson Jumps and Optimal Control
}

\author{
Fathalla A. Rihan, ${ }^{1}$ Chinnathambi Rajivganthi, ${ }^{1}$ and Palanisamy Muthukumar ${ }^{2}$ \\ ${ }^{1}$ Department of Mathematical Sciences, College of Science, UAE University, Al-Ain 15551, UAE \\ ${ }^{2}$ Department of Mathematics, Gandhigram Rural Institute-Deemed University, Gandhigram, Tamil Nadu 624 302, India \\ Correspondence should be addressed to Fathalla A. Rihan; frihan@uaeu.ac.ae
}

Received 18 January 2017; Revised 20 April 2017; Accepted 4 May 2017; Published 15 June 2017

Academic Editor: Thabet Abdeljawad

Copyright (c) 2017 Fathalla A. Rihan et al. This is an open access article distributed under the Creative Commons Attribution License, which permits unrestricted use, distribution, and reproduction in any medium, provided the original work is properly cited.

In this work, we consider a class of fractional stochastic differential system with Hilfer fractional derivative and Poisson jumps in Hilbert space. We study the existence and uniqueness of mild solutions of such a class of fractional stochastic system, using successive approximation theory, stochastic analysis techniques, and fractional calculus. Further, we study the existence of optimal control pairs for the system, using general mild conditions of cost functional. Finally, we provide an example to illustrate the obtained results.

\section{Introduction}

The subject of fractional calculus has gained importance and attractiveness due to its applications in widespread fields of engineering and science. Fractional calculus is successful in describing systems which have long-time memory and long-range interaction [1-3]. Fractional-Order Differential Equations (FODEs) models have been successfully applied in biology systems $[3,4]$, physics $[5,6]$, chemistry and biochemistry [7], hydrology [8], engineering $[9,10]$, medicine [11], finance [12], and control problems [13, 14]. In most cases, the models of FODEs seem to be more regular with the real events compared with integer-order models, because fractional integrals and derivatives allow the explanation of the hereditary and memory properties inherent in various processes and materials $[15,16]$. Many authors described the fractional-order models with the most common definitions of fractional derivatives defined by Caputo and RiemannLiouville sense [17].

Hilfer [5] proposed a general operator for fractional derivative, called "Hilfer fractional derivative," which combines Caputo and Riemann-Liouville fractional derivatives. Hilfer fractional derivative is performed, for example, in the theoretical simulation of dielectric relaxation in glass forming materials. Sandev et al. [18] derived the existence results of fractional diffusion equation with Hilfer fractional derivative which attained in terms of Mittag Leffler functions. Mahmudov and McKibben [19] studied the controllability of fractional dynamical equations with generalized RiemannLiouville fractional derivative by using Schauder fixed point theorem and fractional calculus. Recently, Gu and Trujillo [20] reported the existence results of fractional differential equations with Hilfer derivative based on noncompact measure method. The set of two parameters in "Hilfer fractional derivative" $D_{a^{+}}^{\nu, \mu}$ of order $0 \leq \nu \leq 1$ and $0<\mu<1$ permits one to connect between the Caputo and Riemann-Liouville derivatives $[17,21,22]$. This set of parameters gives an extra degree of freedom on the initial conditions and produces more types of stationary states. Models with Hilfer fractional derivatives are discussed in $[23,24]$.

The deterministic models often fluctuate due to noise. Naturally, the extension of such models is necessary to consider stochastic models, where the related parameters are considered as appropriate Brownian motion and stochastic processes. The modeling of most problems in real situations is described by stochastic differential equations rather than deterministic equations. Thus, it is of great importance to design stochastic effects in the study of fractional-order 
dynamical systems. Chen and Li [25] reported the existence results of fractional stochastic integrodifferential equations with nonlocal initial conditions in Hilbert space. Wang [26] investigated the existence results of fractional stochastic differential equations by using Picard type approximation. Lu and Liu [27] studied, recently, the controllability of fractional stochastic hemivariational inequalities based on multivalued maps and fixed point theorem. The above-mentioned research papers discussed the detail of stochastic differential equations (SDEs) with Brownian motion, Although Brownian motion cannot be used to define the stochastic disturbances in some real systems such as the fluctuations in the financial markets and price dynamics of financial instruments with jumps (see [28]). The authors in [29] studied the existence results of jumps in stock markets and the foreign exchange markets which are based on SDEs with Poisson jumps. Ren et al. [30] reported the existence and stability results of time-dependent stochastic delayed differential equations with Poisson jumps. Recently, Rajivganthi and Muthukumar [31] studied the properties of almost automorphic solutions of fractional stochastic evolution equations with Poisson jumps with the help of solution operator.

To the best of our knowledge, the existence and uniqueness of mild solutions for fractional stochastic differential equations with Hilfer fractional derivative are an untreated topic in the present literature. Herein, we convert the deterministic fractional differential equations into a stochastic fractional differential equation with Hilfer fractional derivative. We then study the existence and uniqueness of mild solutions by using successive approximation. We study the existence and uniqueness of mild solutions by using successive approximation theory. This theory possesses some advantages of linearization for the nonlinear functional with respect to the state variables. We then study the existence of optimal control pairs for the system, using general mild conditions of cost functional.

Consider the fractional stochastic differential equations with Hilfer fractional derivative and Poisson jumps of the form

$$
\begin{aligned}
D_{0^{+}}^{\nu, \mu} x(t)= & A x(t)+f(t, x(t)) \\
& +\int_{0}^{t} g(s, x(s)) d W(s) \\
& +\int_{Z} h(t, x(t), \eta) \widetilde{N}(d t, d \eta), \\
t \in J^{\prime}:=(0, b], & \\
I_{0^{+}}^{(1-\nu)(1-\mu)} x(0)= & x_{0} .
\end{aligned}
$$

Here, $D_{0^{+}}^{\nu, \mu}$ is the Hilfer fractional derivative: $0 \leq v \leq 1,0<$ $\mu<1$ and $J:=[0, b] . A$ is the infinitesimal generator of a strongly continuous semigroup of bounded linear operators $\{T(t)\}_{t \geq 0}$ in Hilbert space $H$. The state variable $x(\cdot)$ is considered in $H$ with a norm $\|$ - \| and an inner product $\langle\cdot, \cdot\rangle$. Let $K$ be another separable Hilbert space and $\{W(t)\}_{t \geq 0}$ is a given $K$-valued Wiener process or Brownian motion with a finite trace nuclear covariance operator $Q \geq 0$. Let $q(\cdot)$ be a Poisson point process in a measurable space $(Z, \mathscr{B}(Z))$ and induced compensating martingale measure $\widetilde{N}(d t, d \eta)$ described on a complete probability space $(\Omega, \mathfrak{F}, P) . f: J \times$ $H \rightarrow H, g: J \times H \rightarrow L_{Q}(K, H)$ and $h: J \times H \times Z \rightarrow H$ are appropriate functions and $L_{\mathrm{Q}}(K, H)$ defines the space of all Q- Hilbert Schmidt operators from $K$ into $H$.

Frequently, the optimal control problems stand up in system engineering. The main goal of optimal control is to find, in an open-loop control, the optimal values of the control variables for the dynamic system which maximize or minimize a given performance index. The determination of optimal control is a difficult task and is open-ended due to the nonlinear nature of dynamic systems. If the FODEs are described in conjunction with a set of initial conditions and performance index, they become Fractional Optimal Control Problems (FOCPs). The FOCP refers to optimization of the performance index subject to dynamical constraints on the control and state which have fractional-order models. There has been some work done in the area of deterministic FOCPs in finite dimensional spaces $[32,33]$ and infinite dimensional cases $[34,35]$. Ren and $\mathrm{Wu}[36]$ discussed the optimal control problem associated with multivalued SDEs with Levy jumps by using Yosida approximation theory. Ahmed [37] studied the existence and optimal control of stochastic initial boundary value problems subject to boundary noise. Rajivganthi et al. [38] investigated the optimal control results of fractional stochastic neutral differential equations in Hilbert space. Motivated by the work done by the authors [20, 35, 38], in this paper, we study additionally the sufficient conditions that guarantee the optimal control results for the fractional stochastic system (1).

This paper is prepared as follows. In Section 2, we provide some remarks, definitions, and lemmas which are useful to prove the main results. Suitable sufficient conditions for existence and uniqueness of (1) are studied in Section 3. Optimal control results are discussed in Section 4. An example is given in Section 5 to verify the theoretical results. We then conclude the paper in the last Section.

\section{Preliminaries}

Let $(\Omega, \mathfrak{F}, P)$ be a complete probability space furnished with complete family of right continuous increasing sub- $\sigma$-algebras $\left\{\mathfrak{F}_{\mathrm{t}}, t \in J\right\}$ satisfying $\mathfrak{F}_{\mathrm{t}} \subset \mathfrak{F}$. We assume that $\mathfrak{F}_{\mathrm{t}}=\sigma(W(s): 0 \leq s \leq t)$ is the $\sigma$-algebra generated by $W$ and $\mathfrak{F}_{\mathrm{t}}=\mathfrak{F}$. Let $\varphi \in L(K, H)$ and define $\|\varphi\|_{Q}^{2}=\operatorname{Tr}\left(\varphi Q \varphi^{*}\right)=\sum_{n=1}^{\infty}\left\|\sqrt{\lambda_{n}} \varphi \zeta_{n}\right\|^{2}$. If $\|\varphi\|_{Q}<\infty$, then $\varphi$ is called a $Q$-Hilbert Schmidt operator. Let $L_{Q}(K, H)$ denote the space of all Q-Hilbert Schmidt operators $\varphi: K \rightarrow H$. The collection of all strongly measurable, square integrable $H$-valued random variables, denoted by $L_{2}(\Omega, \mathfrak{F}, P ; H)=L_{2}(\Omega ; H)$, is a Banach space equipped with norm $\|x(\cdot)\|_{L_{2}}=\left(E\|x(\cdot ; w)\|_{H}^{2}\right)^{1 / 2}$, where the expectation $E$ is defined by $E\left(h_{1}\right)=\int_{\Omega} h_{1}(w) d P$. Let $C\left(J, L_{2}(\Omega ; H)\right)$ be the Banach space of all continuous maps from $J$ into $L_{2}(\Omega ; H)$ satisfying the condition $\sup _{t \in J} E\|x(t)\|^{2}<\infty$. Suppose that $\{q(t): t \in J\}$ is the Poisson point process, taking its value in a measurable space $(Z, \mathscr{B}(Z))$ with a $\sigma$-finite intensity 
measure $\lambda(d \eta)$. The compensating martingale measure and Poisson counting measure are defined by $\widetilde{N}(d s, d \eta)=$ $N(d s, d \eta)-\lambda(d \eta) d s$ and $N(d s, d \eta)$. Let us assume that the filtration $\mathfrak{F}_{t}=\sigma\{N((0, s], A) ; s \leq t, A \in \mathscr{B}(Z)\} \vee \mathcal{N}, t \in J$, produced by $q(\cdot)$ Poisson point process and is augmented, where $\mathcal{N}$ is the class of $P$-null sets.

Define $C^{\nu, \mu}\left(J, L_{2}(\Omega ; H)\right)=\left\{x \in C\left((0, b], L_{2}(\Omega ; H)\right) ;\right.$ $\lim _{t \rightarrow 0^{+}} t^{(1-\gamma)(1-\mu)} x(t)$ exists and its finite $\}$ and let

$$
\|x\|_{\nu, \mu}^{2}=\sup _{0<t \leq b}\left\|t^{(1-v)(1-\mu)} x(t)\right\|^{2} .
$$

Obviously, $C^{\nu, \mu}\left(J, L_{2}(\Omega ; H)\right)$ is a Banach space.

Definition 1. The fractional integral of order $\alpha>0$ with the lower limit $a$ for a function $f:[a, \infty) \rightarrow \mathbb{R}$ is defined as

$$
I_{a^{+}}^{\alpha} f(t)=\frac{1}{\Gamma(\alpha)} \int_{a}^{t} \frac{f(s)}{(t-s)^{1-\alpha}} d s, \quad t>a, \alpha>0,
$$

provided that the right-hand side is pointwise defined on $[0, \infty)$, where $\Gamma$ is the Gamma function.

Definition 2 (see [5]). The Hilfer fractional derivative of order $0 \leq \nu \leq 1$ and $0<\mu<1$ with lower limit $a$ is defined as

$$
D_{a^{+}}^{\nu, \mu} f(t)=I_{a^{+}}^{\nu(1-\mu)} \frac{d}{d t} I_{a^{+}}^{(1-v)(1-\mu)} f(t)
$$

for functions such that the expression on the right-hand side exists.

For more details about the Caputo and RiemannLiouville fractional derivatives, the reader may refer to [22].

Remark 3. When $v=0,0<\mu<1$, the Hilfer fractional derivative coincides with classical Riemann-Liouville fractional derivative:

$$
D_{a^{+}}^{0, \mu} f(t)=\frac{d}{d t} I_{a^{+}}^{1-\mu} f(t)={ }^{L} D_{a^{+}}^{\mu} f(t) .
$$

When $v=1,0<\mu<1$, the Hilfer fractional derivative coincides with classical Caputo fractional derivative:

$$
D_{a^{+}}^{1, \mu} f(t)=I_{a^{+}}^{1-\mu} \frac{d}{d t} f(t)={ }^{C} D_{a^{+}}^{\mu} f(t) .
$$

For $x \in H$, let us define the operators $\left\{S_{\gamma, \mu}(t): t \geq 0\right\}$ and $\left\{P_{\mu}(t): t \geq 0\right\}$ by

$$
\begin{aligned}
S_{\nu, \mu}(t) & =I_{0^{+}}^{\nu(1-\mu)} P_{\mu}(t), \\
P_{\mu}(t) & =t^{\mu-1} T_{\mu}(t), \\
T_{\mu}(t) & =\int_{0}^{\infty} \mu \theta \Psi_{\mu}(\theta) T\left(t^{\mu} \theta\right) d \theta,
\end{aligned}
$$

where $\Psi_{\mu}(\theta)=\sum_{n=1}^{\infty}\left((-\theta)^{n-1} /(n-1) ! \Gamma(1-n \mu)\right) \sin (n \pi \alpha), \theta \epsilon$ $(0, \infty)$, is a function of Wright-type defined on $(0, \infty)$ and verifies $\Psi_{\alpha}(\theta) \geq 0, \int_{0}^{\infty} \Psi_{\alpha}(\theta) d \theta=1, \int_{0}^{\infty} \theta^{\xi} \Psi_{\mu}(\theta) d \theta=\Gamma(1+$ $\xi) / \Gamma(1+\mu \xi), \quad \xi \in(-1, \infty)$, and $\|T(t)\|^{2} \leq M$.
Lemma 4 (see [20]). The operators $S_{\nu, \mu}$ and $P_{\mu}$ have the following properties:

(i) For any fixed $t>0, S_{v, \mu}(t)$ and $P_{\mu}(t)$ are bounded and linear operators, and $\left\|P_{\mu}(t) x\right\|^{2} \leq\left(M t^{2(\mu-1)} /\right.$ $\left.(\Gamma(\mu))^{2}\right)\|x\|^{2}$ and $\left\|S_{\gamma, \mu}(t) x\right\|^{2} \leq\left(M t^{2(\nu-1)(1-\mu)} /(\Gamma(\nu(1-\right.$ $\left.\mu)+\mu))^{2}\right)\|x\|^{2}$.

(ii) $\left\{P_{\mu}(t): t>0\right\}$ is compact, if $\{T(t): t>0\}$ is compact.

Definition 5 (see $[19,20])$. An $H$-valued stochastic process $\left\{x(t) \in C\left(J^{\prime}, L_{2}(\Omega ; H)\right)\right\}$ is a mild solution of system (1) if the process $x$ satisfies the following integral equation:

$$
\begin{gathered}
x(t)=S_{\nu, \mu}(t) x_{0}+\int_{0}^{t} P_{\mu}(t-s) \\
\cdot\left[f(s, x(s))+\int_{0}^{s} g(\tau, x(\tau)) d W(\tau)\right] d s \\
+\int_{0}^{t} \int_{Z} P_{\mu}(t-s) h(s, x(s), \eta) \widetilde{N}(d s, d \eta),
\end{gathered}
$$

$\forall t \in J^{\prime}$.

Remark 6. (i) $D_{0^{+}}^{\nu(1-\mu)} S_{\nu, \mu}(t)=P_{\mu}(t), t \in J^{\prime}$.

(ii) When $\nu=1$, the fractional stochastic equation (1) simplifies to the classical Caputo fractional equation which has been discussed by Chen and $\mathrm{Li}$ [25]. In this case, $S_{1, \mu}(t)=$ $S_{\mu}(t), 0 \leq t \leq b$, where $S_{\mu}(t)$ is defined in [25].

We impose the following assumptions to show the main results:

$\left(H_{1}\right)$ The maps $f: J \times H \rightarrow H, g: J \times H \rightarrow L_{Q}(K, H)$, and $h: J \times H \times Z \rightarrow H$ satisfy, for all $t \in J$ and $x_{1}, x_{2} \in H$,

$$
\left\|f\left(t, x_{1}\right)-f\left(t, x_{2}\right)\right\|^{2} \leq \mathscr{K}\left(\left\|x_{1}-x_{2}\right\|^{2}\right),
$$$$
\int_{0}^{s}\left\|g\left(\tau, x_{1}\right)-g\left(\tau, x_{2}\right)\right\|^{2} d \tau \leq \mathscr{K}\left(\left\|x_{1}-x_{2}\right\|^{2}\right),
$$$$
\int_{Z}\left\|h\left(s, x_{1}, \eta\right)-h\left(s, x_{2}, \eta\right)\right\|^{2} \lambda(d \eta)
$$

$$
\begin{aligned}
& \vee\left(\int_{Z}\left\|h\left(s, x_{1}, \eta\right)-h\left(t, x_{2}, \eta\right)\right\|^{4} \lambda(d \eta)\right)^{1 / 2} \\
& \leq \mathscr{K}\left(\left\|x_{1}-x_{2}\right\|^{2}\right),
\end{aligned}
$$

$$
\left(\int_{Z}\left\|h\left(s, x_{1}, \eta\right)\right\|^{4} \lambda(d \eta)\right)^{1 / 2} \leq \mathscr{K}\left(\left\|x_{1}\right\|^{2}\right),
$$

where $\mathscr{K}(\cdot)$ is a concave nondecreasing function from $\mathbb{R}_{+}$to $\mathbb{R}_{+}$such that $\mathscr{K}(0)=0, \mathscr{K}(\vartheta)>0$ for $\vartheta>0$ and $\int_{0^{+}}(d \vartheta / \mathscr{K}(\vartheta))=+\infty$.

$\left(H_{2}\right)$ For all $t \in J$, there exists a constant $M_{0}>0$ such that

$$
\begin{aligned}
& \|f(t, 0)\|^{2} \vee \int_{0}^{s}\|g(\tau, 0)\|^{2} d \tau \vee \int_{Z}\|h(t, 0, \eta)\|^{2} \lambda(d \eta) \\
& \quad \leq M_{0} .
\end{aligned}
$$


The reader may refer to Remark 2.3 and Lemmas 2.4 and 2.5 in [30], which are useful to prove the main results.
Let the solution $x(t) \in C^{v, \mu}\left(J, L_{2}(\Omega ; H)\right)$ of (1) be defined as follows:

$$
\begin{aligned}
& \lim _{t \rightarrow 0^{+}} t^{(1-\gamma)(1-\mu)} S_{\nu, \mu}(t) x_{0}=\frac{1}{\Gamma(\nu(1-\mu)) \Gamma(\mu)} \int_{0}^{1}(1-s)^{\nu(1-\mu)-1} s^{\mu-1} x_{0} d s=\frac{x_{0}}{\Gamma((\nu(1-\mu)+\mu))}, \\
& t^{(1-\gamma)(1-\mu)} x(t) \\
& = \begin{cases}\frac{x_{0}}{\Gamma((\nu(1-\mu)+\mu))}, & \text { for } t=0, \\
t^{(1-\nu)(1-\mu)} S_{\nu, \mu}(t) x_{0}+t^{(1-\nu)(1-\mu)} \int_{0}^{t} P_{\mu}(t-s)\left[f(s, x(s))+\int_{0}^{s} g(\tau, x(\tau)) d W(\tau)\right] d s+t^{(1-\gamma)(1-\mu)} \int_{0}^{t} \int_{Z} P_{\mu}(t-s) h(s, x(s), \eta) \widetilde{N}(d s, d \eta), & \text { for } 0<t \leq b .\end{cases}
\end{aligned}
$$

We refer to $[25,38,39]$ for further discussion of stochastic concepts.

\section{Existence and Uniqueness of Mild Solutions}

In order to prove the existence of mild solution for system (1), let us consider the sequence of successive approximations defined as follows:

$$
\begin{aligned}
& x^{0}(t)=t^{(1-\nu)(1-\mu)} S_{\nu, \mu}(t) x_{0}, \quad 0<t \leq b, \\
& x^{n}(t)=\frac{x_{0}}{\Gamma((\nu(1-\mu)+\mu))}, \quad t=0, n=1,2, \ldots, \\
& x^{n}(t)=t^{(1-v)(1-\mu)} S_{\nu, \mu}(t) x_{0}+t^{(1-\nu)(1-\mu)} \int_{0}^{t} P_{\mu}(t-s) \\
& \cdot {\left[f\left(s, x^{n-1}(s)\right)+\int_{0}^{s} g\left(\tau, x^{n-1}(\tau)\right) d W(\tau)\right] d s } \\
&+ t^{(1-v)(1-\mu)} \int_{0}^{t} \int_{Z} P_{\mu}(t-s) h\left(s, x^{n-1}(s), \eta\right) \\
& \cdot \widetilde{N}(d s, d \eta), \quad 0<t \leq b, n=1,2, \ldots
\end{aligned}
$$

Theorem 7. If the assumptions $\left(H_{1}\right)-\left(H_{2}\right)$ are satisfied, then system (1) has a unique mild solution in the space $C^{\nu, \mu}\left(J, L_{2}(\Omega ; H)\right)$, provided that $\left(3 M /(\Gamma(\mu))^{2}\right) t^{2(1-\nu)(1-\mu)}(b+$ $\operatorname{Tr}(Q)+2 C)<1$, with $1 / 2<\mu<1$ and $t \in J$.

Proof. For better readability, we break the proof into a sequence of steps.

Step 1. For all $t \in J$, the sequence $x^{n}(t), n \geq 1$, is bounded.

It is obvious that $x^{0}(t) \in C^{\nu, \mu}\left(J, L_{2}(\Omega ; H)\right)$. Let $x^{0}$ be a fixed initial approximation to (12). Let us use the assumptions $\left(H_{1}\right)$ and $\left(H_{2}\right)$, Holder inequality, Doob Martingale inequality and Burkholder-Davis-Gundy inequality for pure jump stochastic integral in $H$ ([30]). We have

$$
\begin{gathered}
E\left\|x^{n}(t)\right\|^{2} \leq \frac{4 M\left\|x_{0}\right\|^{2}}{(\Gamma(\nu(1-\mu)+\mu))^{2}}+\frac{4 M b t^{2(1-\nu)(1-\mu)}}{(\Gamma(\mu))^{2}} \\
\cdot \int_{0}^{t}(t-s)^{2(\mu-1)} E\left\|f\left(s, x^{n-1}(s)\right)\right\|^{2} d s
\end{gathered}
$$

$$
\begin{aligned}
& +\frac{4 M \operatorname{Tr}(Q) t^{2(1-\nu)(1-\mu)}}{(\Gamma(\mu))^{2}} \int_{0}^{t}(t-s)^{2(\mu-1)} \\
& \cdot\left(\int_{0}^{s} E\left\|g\left(\tau, x^{n-1}(\tau)\right)\right\|^{2} d \tau\right) d s \\
& +\frac{4 M C t^{2(1-\nu)(1-\mu)}}{(\Gamma(\mu))^{2}}\left[\int_{0}^{t}(t-s)^{2(\mu-1)}\right. \\
& \cdot \int_{Z} E\left\|h\left(s, x^{n-1}(s), \eta\right)\right\|^{2} \lambda(d \eta) d s+\int_{0}^{t}(t \\
& -s)^{2(\mu-1)} \\
& \left.\cdot\left(\int_{Z} E\left\|h\left(s, x^{n-1}(s), \eta\right)\right\|^{4} \lambda(d \eta)\right)^{1 / 2} d s\right]
\end{aligned}
$$$$
\leq \frac{4 M\left\|x_{0}\right\|^{2}}{(\Gamma(\nu(1-\mu)+\mu))^{2}}+\frac{4 M b t^{2(1-\nu)(1-\mu)}}{(\Gamma(\mu))^{2}} \int_{0}^{t}(t
$$$$
-s)^{2(\mu-1)}\left[E\left\|f\left(s, x^{n-1}(s)\right)-f(s, 0)\right\|^{2}\right.
$$$$
\left.+E\|f(s, 0)\|^{2}\right] d s+\frac{4 M \operatorname{Tr}(Q) t^{2(1-\nu)(1-\mu)}}{(\Gamma(\mu))^{2}}
$$$$
\cdot \int_{0}^{t}(t-s)^{2(\mu-1)}
$$$$
\cdot\left(\int_{0}^{s} E\left\|g\left(\tau, x^{n-1}(\tau)\right)-g(\tau, 0)\right\|^{2} d \tau\right.
$$$$
\left.+\int_{0}^{s} E\|g(\tau, 0)\|^{2} d \tau\right) d s
$$$$
+\frac{4 M C t^{2(1-v)(1-\mu)}}{(\Gamma(\mu))^{2}}\left[\int_{0}^{t}(t-s)^{2(\mu-1)}\right.
$$$$
\cdot\left(\int_{Z} E\left\|h\left(s, x^{n-1}(s), \eta\right)-h(s, 0, \eta)\right\|^{2}\right.
$$$$
\left.+E\|h(s, 0, \eta)\|^{2}\right) \lambda(d \eta) d s+\int_{0}^{t}(t-s)^{2(\mu-1)}
$$$$
\left.\cdot\left(\int_{Z} E\left\|h\left(s, x^{n-1}(s), \eta\right)\right\|^{4} \lambda(d \eta)\right)^{1 / 2} d s\right]
$$ 


$$
\begin{aligned}
& \leq \frac{4 M\left\|x_{0}\right\|^{2}}{(\Gamma(\nu(1-\mu)+\mu))^{2}}+\frac{4 M t^{2(1-\nu)(1-\mu)}}{(\Gamma(\mu))^{2}}(b \\
& +\operatorname{Tr}(Q)+C) M_{0} \frac{b^{2 \mu-1}}{2 \mu-1}+\frac{4 M t^{2(1-\nu)(1-\mu)}}{(\Gamma(\mu))^{2}}(b \\
& +\operatorname{Tr}(Q)+2 C) \int_{0}^{t}(t-s)^{2(\mu-1)} \\
& \cdot \mathscr{K}\left(E\left\|x^{n-1}(s)\right\|^{2}\right) d s,
\end{aligned}
$$

where $M_{1}=4 M\left\|x_{0}\right\|^{2} /(\Gamma(\nu(1-\mu)+\mu))^{2}+\left(4 M t^{2(1-\nu)(1-\mu)} /\right.$ $\left.(\Gamma(\mu))^{2}\right)(b+\operatorname{Tr}(Q)+C) M_{0}\left(b^{2 \mu-1} /(2 \mu-1)\right)$ and $C>0$ is constant. Here, $\mathscr{K}(\cdot)$ is concave and $\mathscr{K}(0)=0$, and one can find a pair of positive constants $a_{1}$ and $a_{2}$ such that $\mathscr{K}(t) \leq$ $a_{1}+a_{2} t$, for $t \geq 0$. Then

$$
\begin{gathered}
E\left\|x^{n}(t)\right\|^{2} \leq M_{1}+\frac{4 M t^{2(1-\nu)(1-\mu)}}{(\Gamma(\mu))^{2}}(b+\operatorname{Tr}(Q)+2 C) \\
\cdot a_{1} \frac{b^{2 \mu-1}}{2 \mu-1}+\frac{4 M t^{2(1-\nu)(1-\mu)}}{(\Gamma(\mu))^{2}}(b+\operatorname{Tr}(Q)+2 C) \\
\cdot a_{2} \int_{0}^{t}(t-s)^{2(\mu-1)} E\left\|x^{n-1}(s)\right\|^{2} d s \leq M_{2} \\
+\frac{4 M t^{2(1-\nu)(1-\mu)}}{(\Gamma(\mu))^{2}}(b+\operatorname{Tr}(Q)+2 C) \\
\cdot a_{2} \int_{0}^{t}(t-s)^{2(\mu-1)} E\left\|x^{n-1}(s)\right\|^{2} d s,
\end{gathered}
$$

where

$$
\begin{aligned}
M_{2}= & M_{1} \\
& +\frac{4 M t^{2(1-v)(1-\mu)}}{(\Gamma(\mu))^{2}}(b+\operatorname{Tr}(Q)+2 C) a_{1} \frac{b^{2 \mu-1}}{2 \mu-1} .
\end{aligned}
$$

For any $k \geq 1$,

$$
\begin{aligned}
& \max _{1 \leq n \leq k} E \sup _{0 \leq s \leq t}\left\|x^{n-1}(s)\right\|^{2} \leq E\left\|x^{0}(s)\right\|^{2}+\max _{1 \leq n \leq k} E \\
& \cdot \sup _{0 \leq s \leq t}\left\|x^{n}(s)\right\|^{2}, \\
& \max _{1 \leq n \leq k} E \sup _{0 \leq s \leq t}\left\|x^{n}(s)\right\|^{2} \leq M_{2} \\
& +\frac{4 M t^{2(1-v)(1-\mu)}}{(\Gamma(\mu))^{2}}(b+\operatorname{Tr}(Q)+2 C) \\
& \cdot a_{2} \int_{0}^{t}(t-s)^{2(\mu-1)} E\left\|x^{0}(s)\right\|^{2} d s \\
& +\frac{4 M t^{2(1-\nu)(1-\mu)}}{(\Gamma(\mu))^{2}}(b+\operatorname{Tr}(Q)+2 C)
\end{aligned}
$$

$$
\begin{aligned}
& \cdot a_{2} \int_{0}^{t}(t-s)^{2(\mu-1)} \max _{1 \leq n \leq k} E \sup _{0 \leq r \leq s}\left\|x^{n}(r)\right\|^{2} d s \leq M_{3} \\
& +M_{4} \int_{0}^{t}(t-s)^{2(\mu-1)} E\left\|x^{n}(s)\right\|^{2} d s \leq M_{3} \\
& \cdot \exp \left(\frac{M_{4} b^{2 \mu-1}}{2 \mu-1}\right)
\end{aligned}
$$

where $M_{3}=M_{2}+\left(4 M t^{2(1-\nu)(1-\mu)} /(\Gamma(\mu))^{2}\right)(b+\operatorname{Tr}(Q)+$ 2C) $a_{2} \int_{0}^{t}(t-s)^{2(\mu-1)} E\left\|x^{0}(s)\right\|^{2} d s<\infty$ and $M_{4}=$ $\left(4 M t^{2(1-v)(1-\mu)} /(\Gamma(\mu))^{2}\right)(b+\operatorname{Tr}(Q)+2 C) a_{2}$. Thus $E\left\|x^{n}(t)\right\|^{2}<$ $\infty$, for $n \geq 1, t \in J$, which shows that the sequence $x^{n}(t), n \geq$ 1 , is bounded in $C^{\nu, \mu}\left(J, L_{2}(\Omega ; H)\right)$.

Step 2. Sequence $x^{n}(t), n \geq 1$, is a Cauchy sequence.

From (12), for all $n \geq 1$ and $0 \leq t \leq b$,

$$
\begin{aligned}
E\left\|x^{n+1}(t)-x^{n}(t)\right\|^{2}=E \| t^{(1-v)(1-\mu)} \int_{0}^{t} P_{\mu}(t-s) & \\
\cdot & {\left[\left(f\left(s, x^{n}(s)\right)-f\left(s, x^{n-1}(s)\right)\right)\right.} \\
+ & \left.\left(\int_{0}^{s} g\left(\tau, x^{n}(\tau)\right) d W(\tau)-\int_{0}^{s} g\left(\tau, x^{n-1}(\tau)\right) d W(\tau)\right)\right] d s \\
+ & \int_{0}^{t} \int_{Z} P_{\mu}(t-s)\left(h\left(s, x^{n}(s), \eta\right)-h\left(s, x^{n-1}(s), \eta\right)\right) \\
\cdot & \widetilde{N}(d s, d \eta) \|^{2} \leq \frac{3 M}{(\Gamma(\mu))^{2}} t^{2(1-v)(1-\mu)}(b+\operatorname{Tr}(Q)+2 C) \int_{0}^{t}(t \\
& -s)^{2(\mu-1)} \mathscr{K}\left(E\left\|x^{n}(s)-x^{n-1}(s)\right\|^{2}\right) d s .
\end{aligned}
$$

Let $\Phi_{n}(t)=\sup _{t \in[0, b]} E\left\|x^{n+1}(t)-x^{n}(t)\right\|^{2}$. Thus, we have in the above inequality that

$$
\begin{gathered}
\Phi_{n}(t) \leq \frac{3 M}{(\Gamma(\mu))^{2}} t^{2(1-\nu)(1-\mu)}(b+\operatorname{Tr}(Q)+2 C) \\
\cdot \int_{0}^{t}(t-s)^{2(\mu-1)} \mathscr{K}\left(E\left\|x^{n}(s)-x^{n-1}(s)\right\|^{2}\right) d s, \\
\Phi_{n}(t) \leq \frac{3 M}{(\Gamma(\mu))^{2}} t^{2(1-v)(1-\mu)}(b+\operatorname{Tr}(Q)+2 C) \\
\cdot \int_{0}^{t}(t-s)^{2(\mu-1)} \mathscr{K}\left(\Phi_{n-1}(s)\right) d s, \quad 0 \leq t \leq b .
\end{gathered}
$$

Choose $b_{1} \in[0, b]$ such that

$$
\begin{aligned}
& M_{5} \int_{0}^{t}(t-s)^{2(\mu-1)} \mathscr{K}\left(\Phi_{n-1}(s)\right) d s \\
& \quad \leq M_{5} \int_{0}^{t}(t-s)^{2(\mu-1)} \Phi_{n-1}(s) d s
\end{aligned}
$$

$$
0 \leq t \leq b_{1}, n \geq 1 \text {. }
$$


Moreover,

$$
\begin{gathered}
E\left\|x^{1}(t)-x^{0}(t)\right\|^{2} \leq \frac{3 M}{(\Gamma(\mu))^{2}} \\
\cdot t^{2(1-v)(1-\mu)}(b+\operatorname{Tr}(Q)+2 C) \\
\cdot \int_{0}^{t}(t-s)^{2(\mu-1)} \mathscr{K}\left(E\left\|x^{0}(s)\right\|^{2}\right) d s .
\end{gathered}
$$

We take the supreme over $t$ and use $\Phi_{n}$ :

$$
\begin{gathered}
\Phi_{0}(t)=\sup _{t \in[0, b]} E\left\|x^{1}(t)-x^{0}(t)\right\|^{2} \leq \frac{3 M}{(\Gamma(\mu))^{2}} \\
\cdot t^{2(1-\nu)(1-\mu)}(b+\operatorname{Tr}(Q)+2 C) \\
\cdot \int_{0}^{t}(t-s)^{2(\mu-1)} \mathscr{K}\left(E\left\|x^{0}(s)\right\|^{2}\right) d s=C_{1} .
\end{gathered}
$$

Now, for $n=1$ in (18), we have

$$
\begin{aligned}
& \Phi_{1}(t) \leq \frac{3 M}{(\Gamma(\mu))^{2}} t^{2(1-v)(1-\mu)}(b+\operatorname{Tr}(Q)+2 C) \\
& \cdot \int_{0}^{t}(t-s)^{2(\mu-1)} \mathscr{K}\left(\Phi_{0}(s)\right) d s \\
& \quad \leq M_{5} \int_{0}^{t}(t-s)^{2(\mu-1)} \mathscr{K}\left(\Phi_{0}(s)\right) d s \\
& \quad \leq M_{5} \int_{0}^{t}(t-s)^{2(\mu-1)} \Phi_{0}(s) d s \\
& \quad \leq M_{5} \int_{0}^{t}(t-s)^{2(\mu-1)} C_{1} d s \leq M_{5} C_{1} \frac{b^{2(\mu-1)+1}}{2(\mu-1)+1} .
\end{aligned}
$$

And, for $n=2$ in (18), we have

$$
\begin{aligned}
\Phi_{2}(t) & \leq M_{5} \int_{0}^{t}(t-s)^{2(\mu-1)} \mathscr{K}\left(\Phi_{1}(s)\right) d s \\
& \leq M_{5} \int_{0}^{t}(t-s)^{2(\mu-1)} \Phi_{1}(s) d s \\
& \leq M_{5} \int_{0}^{t} M_{5} C_{1} \frac{b^{2(\mu-1)+1}}{2(\mu-1)+1} d s \\
& \leq C_{1} \frac{b^{2(\mu-1)}}{2(\mu-1)+1}\left(M_{5}\right)^{2} \frac{b^{2}}{2 !} .
\end{aligned}
$$

By applying mathematical induction in (18) and with the above work, we have

$$
\Phi_{n}(t) \leq C_{1} \frac{b^{2(\mu-1)}}{2(\mu-1)+1}\left(M_{5}\right)^{n} \frac{b^{n}}{n !},
$$

So, for any $m \geq n \geq 0$,

$$
\begin{aligned}
& \sup _{t \in\left[0, b_{1}\right]} E\left\|x^{m}(t)-x^{n}(t)\right\|^{2} \\
& \leq \sum_{r=n}^{+\infty} \sup _{t \in\left[0, b_{1}\right]} E\left\|x^{r+1}(t)-x^{r}(t)\right\|^{2} \\
& \leq \sum_{r=n}^{+\infty} C_{1} \frac{b^{2(\mu-1)}}{2(\mu-1)+1}\left(M_{5}\right)^{r} \frac{b^{r}}{r !} \longrightarrow 0,
\end{aligned}
$$

as $n \longrightarrow \infty$.

Step 3. The existence and uniqueness of solution for system (1) are tackled as follows.

The Borel-Cantelli Lemma says that $x^{n}(t) \rightarrow x(t)$, as $n \rightarrow$ $\infty$ uniformly for $0 \leq t \leq b$. Thus, for all $t \in J$, taking limits on both sides of (12), one can obtain that $x(t)$ is a solution to (1). Next, to show the uniqueness, let $x_{1}, x_{2} \in C^{\nu, \mu}\left(J, L_{2}(\Omega ; H)\right)$ be two solutions on $t \in J$. For $t \in J$,

$$
\begin{aligned}
E \| & x_{1}(t)-x_{2}(t) \|^{2} \\
& \leq \frac{3 M t^{2(1-v)(1-\mu)}}{(\Gamma(\mu))^{2}}(b+\operatorname{Tr}(Q)+2 C) \\
& \cdot \int_{0}^{t}(t-s)^{2(\mu-1)} \mathscr{K}\left(E\left\|x_{1}(s)-x_{2}(s)\right\|^{2}\right) d s .
\end{aligned}
$$

Thus, from Bihari inequality, it yields that

$$
\sup _{t \in J} E\left\|x_{1}(t)-x_{2}(t)\right\|^{2}=0 \text {. }
$$

Therefore, $x_{1}(t)=x_{2}(t)$, for all $t \in J$.

\section{Optimal Control Results}

Let $Y$ be reflexive Banach space in which controls $u$ take values. Let us denote a class of nonempty convex and closed subsets of $Y$ by $2^{Y} \backslash\{\varnothing\}$. The multivalued function $v: J \rightarrow$ $2^{Y} \backslash\{\varnothing\}$ is measurable and $v(\cdot) \subset \mathscr{E}$, where $\mathscr{E}$ is a bounded set of $Y$. The admissible control set $U_{\text {ad }}=\left\{u(\cdot) \in L_{2}(\mathscr{E})\right.$ | $u(t) \in v(t)$.a.e $\}$. Then $U_{\mathrm{ad}} \neq \varnothing$ and $U_{\mathrm{ad}} \subset L_{2}(J, Y)$ is bounded, closed, and convex [35]. The fractional stochastic control problem with Hilfer fractional derivative is of the form

$$
\begin{aligned}
D_{0^{+}}^{\nu, \mu} x(t)= & A x(t)+B(t) u(t)+f(t, x(t)) \\
& +\int_{0}^{t} g(s, x(s)) d W(s) \\
& +\int_{Z} h(t, x(t), \eta) \widetilde{N}(d t, d \eta),
\end{aligned}
$$

$t \in J^{\prime}$

$I_{0^{+}}^{(1-\nu)(1-\mu)} x(0)=x_{0}$ 
By using Definition 5 for every $u \in U_{\mathrm{ad}}$, there exists $b>0$, and the solution of the control problem (28) is defined as

$$
\begin{gathered}
x(t)=S_{\gamma, \mu}(t) x_{0}+\int_{0}^{t} P_{\mu}(t-s)[B(s) u(s) \\
\left.+f(s, x(s))+\int_{0}^{s} g(\tau, x(\tau)) d W(\tau)\right] d s \\
+\int_{0}^{t} \int_{Z} P_{\mu}(t-s) h(s, x(s), \eta) \widetilde{N}(d s, d \eta),
\end{gathered}
$$

$\forall t \in J$

$\left(H_{3}\right)$ The operator $B \in L_{2}(J, L(Y, H))$; $\|B\|_{2}$ denotes the norm of operator $B$ in Banach space $L_{2}(J, L(Y, H))$. Obliviously, $B u \in L_{2}(J, H)$ for every $u \in U_{\text {ad }}$.

Lemma 8. Let $\left(H_{1}\right)-\left(H_{3}\right)$ hold. If system (28) is mildly solvable on $J$ with respect to $u \in U_{a d}$ and $1 / 2<\mu<1$, then there exists a constant $\rho>0$ such that $E\|x(t)\|^{2} \leq \rho$ for all $t \in J$.

Proof. If $x$ is a mild solution of (28) with respect to $u \in U_{\mathrm{ad}}$, then $x$ satisfies equation (29). Using hypotheses $\left(H_{1}\right)-\left(H_{3}\right)$, as well as Burkholder-Davis-Gundy inequality ([30]), we obtain

$$
\begin{aligned}
& E\|x(t)\|^{2} \leq 5 E\left\|S_{\nu, \mu}(t) x_{0}\right\|^{2}+5 b \int_{0}^{t}\left\|P_{\mu}(t-s)\right\|^{2} \\
& \cdot\|B\|^{2}\|u(s)\|^{2} d s+5 b \int_{0}^{t}\left\|P_{\mu}(t-s)\right\|^{2} \\
& \cdot E\|f(s, x(s))\|^{2} d s+5 \operatorname{Tr}(Q) \int_{0}^{t}\left\|P_{\mu}(t-s)\right\|^{2} \\
& \cdot \int_{0}^{s} E\|g(\tau, x(\tau))\|^{2} d \tau d s+5 C \int_{0}^{t} \| P_{\mu}(t \\
& -s) \|^{2}\left(\int_{Z} E\|h(s, x(s), \eta)\|^{2} \lambda d(\eta) d s\right. \\
& \left.+\left(\int_{Z} E\|h(s, x(s), \eta)\|^{4} \lambda d(\eta)\right)^{1 / 2} d s\right) \\
& \left.+\int_{0}^{\tau} E\|g(\tau, 0)\|^{2} d \tau\right) d s+\frac{5 M C}{(\Gamma(\mu))^{2}}\left\{\int_{0}^{t}(t\right. \\
& +\frac{5 M \operatorname{Tr}(Q)}{(\Gamma(\mu))^{2}(t-s)^{2(\mu-1)}} \\
& +\left(\int_{0}^{\tau} E\|g(\tau, x(\tau))-g(\tau, 0)\|^{2} d \tau\right. \\
& +s)^{2(\mu-1)}\|u(s)\|^{2} d s+\frac{5 M b}{(\Gamma(\mu))^{2}} \int_{0}^{t}(t-s)^{2(\mu-1)} \\
& +(1-\mu)+\mu))^{2}\left\|x_{0}\right\|^{2}+\frac{5 M b\|B\|^{2}}{(\Gamma(\mu))^{2}}(t
\end{aligned}
$$

$$
\begin{aligned}
& -s)^{2(\mu-1)}\left(\int_{Z} E\|h(s, x(s), \eta)-h(s, 0, \eta)\|^{2}\right. \\
& \left.\left.+E\|h(s, 0, \eta)\|^{2}\right) \lambda d(\eta) d s\right\}+\frac{5 M C}{(\Gamma(\mu))^{2}} \int_{0}^{t}(t \\
& -s)^{2(\mu-1)}\left(\int_{Z} E\|h(s, x(s), \eta)\|^{4} \lambda d(\eta)\right)^{1 / 2} d s \\
& \leq \frac{5 M b^{2(\nu-1)(1-\mu)}}{(\Gamma(\nu(1-\mu)+\mu))^{2}}\left\|x_{0}\right\|^{2}+\frac{5 M b\|B\|^{2}}{(\Gamma(\mu))^{2}}\left(\int_{0}^{t}(t\right.
\end{aligned}
$$$$
\begin{aligned}
& \left.-s)^{2(\mu-1)(p /(p-1))} d s\right)^{(p-1) / p} \\
& \cdot\left(\int_{0}^{t}\|u(s)\|^{2 p} d s\right)^{1 / p}+\frac{5 M M_{0}(b+\operatorname{Tr}(Q)+C)}{(\Gamma(\mu))^{2}}
\end{aligned}
$$$$
\cdot \frac{b^{2 \mu-1}}{2 \mu-1}+\frac{5 M(b+\operatorname{Tr}(Q)+2 C)}{(\Gamma(\mu))^{2}} \int_{0}^{t}(t-s)^{2(\mu-1)}
$$$$
\cdot \mathscr{K}\left(E\|x(s)\|^{2}\right) d s,
$$

where $M_{6}=\left(5 M b^{2(\nu-1)(1-\mu)} /(\Gamma(\nu(1-\mu)+\mu))^{2}\right)\left\|x_{0}\right\|^{2}$ $+\left(5 M b\|B\|^{2} /(\Gamma(\mu))^{2}\right)\left(b^{(2 \mu p-p-1) /(p-1)} /((2 \mu p-p-1) /(p\right.$ $-1)))^{(p-1) / p}\|u\|_{L_{p}(J, Y)}^{2}+\left(5 M M_{0}(b+\operatorname{Tr}(Q)+C) /(\Gamma(\mu))^{2}\right)\left(b^{2 \mu-1} /\right.$ $(2 \mu-1))$ and $M_{7}=5 M(b+\operatorname{Tr}(Q)+2 C) /(\Gamma(\mu))^{2}$. Given that $\mathscr{K}(\cdot)$ is concave and $\mathscr{K}(0)=0$, one can find a pair of positive constants $a_{1}$ and $a_{2}$ such that $\mathscr{K}(t) \leq a_{1}+a_{2} t$, for $t \geq 0$. Then

$$
\begin{aligned}
E\|x(t)\|^{2} \leq & M_{6}+M_{7} a_{1} \frac{b^{2 \mu-1}}{2 \mu-1} \\
& +M_{7} a_{2} \int_{0}^{t}(t-s)^{2(\mu-1)} E\|x(s)\|^{2} d s .
\end{aligned}
$$

By using Gronwall's inequality,

$$
\begin{aligned}
E\|x(t)\|^{2} & \\
& \leq\left(M_{6}+M_{7} a_{1} \frac{b^{2 \mu-1}}{2 \mu-1}\right) \exp \left(M_{7} a_{2} \frac{b^{2 \mu-1}}{2 \mu-1}\right)=\rho \\
& <\infty .
\end{aligned}
$$

Theorem 9 (see [35]). Under hypotheses $\left(H_{1}\right)-\left(H_{3}\right)$ and for each $u \in U_{a d}$, system (28) is mildly solvable on $J$ and the solution is unique.

Minimize a performance index of the following form:

$$
\mathfrak{J}(x, u)=\int_{0}^{b} \mathscr{L}\left(t, x^{u}(t), u(t)\right) d t
$$


among all the admissible state control pairs of system (28); that is, find an admissible state control pair $\left(x^{0}, u^{0}\right) \in$ $C\left(J, L_{2}(\Omega ; H)\right) \times U_{a d}$ such that

$$
\mathfrak{J}\left(x^{0}, u^{0}\right) \leq \mathfrak{J}(x, u) \quad \forall u \in U_{a d} ;
$$

here $x^{u}(t)$ defines the mild solution of (28) corresponding to $u \in U_{a d}$. Assume that

$\left(H_{4}\right)$ the cost functional $\mathscr{L}: J \times H \times Y \rightarrow \mathbb{R} \cup\{+\infty\}$ is such that

(i) $(t, x, u) \rightarrow \mathscr{L}(t, x, u)$ is measurable,

(ii) $\mathscr{L}(t, \cdot, \cdot)$ is lower semicontinuous on $H \times Y$ for almost all $t \in J$,

(iii) $\mathscr{L}(t, x, \cdot)$ is convex on $Y$ for all $x \in H$ and almost all $t \in J$,

(iv) there exist constants $d \geq 0, j>0, \rho_{1} \geq 0$, and $\rho_{1} \in L_{1}(J, \mathbb{R})$ such that

$$
\mathscr{L}(t, x(t), u(t)) \geq \rho_{1}(t)+d\|x\|_{H}+j\|u\|_{Y}^{2} .
$$

Theorem 10. If $B$ is strongly continuous operator, hypotheses $\left(H_{1}\right)-\left(H_{4}\right)$ and Theorem 9 hold. Then the stochastic control problem (28) permits at least one optimal control pair.

Proof. The main aim is to minimize the value of $\mathfrak{\Im}(x, u)$. If $\inf _{(x, u) \in \mathscr{A}_{\text {ad }}} \mathfrak{J}(x, u)=+\infty,\left(\mathscr{A}_{\mathrm{ad}}=\{(x, u)\right.$ such that $x$ is a mild solution of (28) with $\left.\left.u \in U_{\mathrm{ad}}\right\}\right)$; then there is nothing to prove. Assume that $\inf _{(x, u) \in \mathscr{A} \text { ad }} \mathfrak{\Im}(x, u)=\epsilon<\infty$. Using $\left(H_{4}\right)$, we have $\epsilon>-\infty$. By definition of infimum, there exists a minimizing sequence feasible pair $\left\{\left(x_{n}, u_{n}\right)\right\}_{n \geq 1} \subset \mathscr{A}_{\text {ad }}$, such that $\mathfrak{\Im}\left(x_{n}, u_{n}\right) \rightarrow \epsilon$ as $n \rightarrow+\infty$. Since $u_{n} \in U_{\text {ad }}$, $\left\{u_{n}\right\}_{n \geq 1} \subset L_{2}(J, Y)$ is bounded. Thus, there exists $\widehat{u} \in L_{2}(J, Y)$ and a subsequence extracted from $\left(u_{n}\right)$ (still called $\left(u_{n}\right)$ ) such that $u_{n} \rightarrow \widehat{u}$ weakly in $L_{2}(J, Y)$. Moreover, from the convexity and closeness of $U_{\mathrm{ad}}$ and Mazur's Theorem, we know that $\widehat{u} \in U_{\text {ad }}$. Suppose that $x_{n}$ and $\widehat{x}$ are the mild solutions of (28) corresponding to $u_{n}$ and $\widehat{u}$, respectively. $x_{n}$ and $\widehat{x}$ satisfy the following equations, respectively:

$$
\begin{gathered}
x_{n}(t)=S_{v, \mu}(t) x_{0}+\int_{0}^{t} P_{\mu}(t-s)\left[B(s) u_{n}(s)\right. \\
\left.+f\left(s, x_{n}(s)\right)+\int_{0}^{s} g\left(\tau, x_{n}(\tau)\right) d W(\tau)\right] d s \\
+\int_{0}^{t} \int_{Z} P_{\mu}(t-s) h\left(s, x_{n}(s), \eta\right) \widetilde{N}(d s, d \eta), \\
\widehat{x}(t)=S_{v, \mu}(t) x_{0}+\int_{0}^{t} P_{\mu}(t-s)[B(s) \widehat{u}(s) \\
\left.+f(s, \widehat{x}(s))+\int_{0}^{s} g(\tau, \widehat{x}(\tau)) d W(\tau)\right] d s \\
+\int_{0}^{t} \int_{Z} P_{\mu}(t-s) h(s, \widehat{x}(s), \eta) \widetilde{N}(d s, d \eta) .
\end{gathered}
$$

From the boundedness of $u_{n}$ and $\widehat{u}$, Lemma 8 , one can verify that there exists a positive number $\rho$ such that $\left\|x_{n}\right\|,\|\widehat{x}\| \leq \rho$. Then, for $t \in J,(p+1) / 2 p<\mu<1$.

$$
\begin{aligned}
& E\left\|x_{n}(t)-\widehat{x}(t)\right\|^{2}=E \| \int_{0}^{t} P_{\mu}(t-s) \\
& \cdot\left[\left(f\left(s, x_{n}(s)\right)-f(s, \widehat{x}(s))\right)+\left(B(s) u_{n}(s)-B(s) \widehat{u}(s)\right)\right. \\
& \left.+\left(\int_{0}^{s} g\left(\tau, x_{n}(\tau)\right) d W(\tau)-\int_{0}^{s} g(\tau, \widehat{x}(\tau)) d W(\tau)\right)\right] d s \\
& +\int_{0}^{t} \int_{Z} P_{\mu}(t-s)\left(h\left(s, x_{n}(s), \eta\right)-h(s, \widehat{x}(s), \eta)\right) \\
& \cdot \widetilde{N}(d s, d \eta) \|^{2} \leq \frac{4 M}{(\Gamma(\mu))^{2}}(b+\operatorname{Tr}(Q)+2 C) \int_{0}^{t}(t-s)^{2(\mu-1)} \\
& \cdot \mathscr{K}\left(E\left\|x_{n}(s)-\widehat{x}(s)\right\|^{2}\right) d s+\frac{4 M b}{(\Gamma(\mu))^{2}} \int_{0}^{t}(t-s)^{2(\mu-1)} \\
& \cdot E\left\|B(s) u_{n}(s)-B(s) \widehat{u}(s)\right\|^{2} d s \leq \frac{4 M}{(\Gamma(\mu))^{2}}(b \\
& +\operatorname{Tr}(Q)+2 C) \int_{0}^{t}(t-s)^{2(\mu-1)} \mathscr{K}\left(E\left\|x_{n}(s)-\widehat{x}(s)\right\|^{2}\right) d s \\
& +\frac{4 M b}{(\Gamma(\mu))^{2}}\left(\int_{0}^{t}(t-s)^{2(\mu-1)(p /(p-1))} d s\right)^{(p-1) / p}\left(\int_{0}^{t} E \| B(s)\right. \\
& \left.\cdot u_{n}(s)-B(s) \widehat{u}(s) \|^{2 p} d s\right)^{1 / p} \leq \frac{4 M}{(\Gamma(\mu))^{2}}(b+\operatorname{Tr}(Q) \\
& +2 C) \int_{0}^{t}(t-s)^{2(\mu-1)} \mathscr{K}\left(E\left\|x_{n}(s)-\widehat{x}(s)\right\|^{2}\right) d s \\
& +\frac{4 M b}{(\Gamma(\mu))^{2}}\left((p-1) \frac{b^{(2 \mu p-p-1) /(p-1)}}{2 \mu p-p-1}\right)^{(p-1) / p}\left(\int_{0}^{b} E \| B(s)\right. \\
& \left.\cdot u_{n}(s)-B(s) \widehat{u}(s) \|^{2 p} d s\right)^{1 / p} \cdot
\end{aligned}
$$

Using the continuous operator $B$ and Lebesgue's dominated convergence theorem, we have

$$
\begin{aligned}
\frac{4 M}{(\Gamma(\mu))^{2}}(b+\operatorname{Tr}(Q)+2 C) & \\
\cdot \int_{0}^{t}(t-s)^{2(\mu-1)} \mathscr{K}\left(E\left\|x_{n}(s)-\widehat{x}(s)\right\|^{2}\right) d s & \longrightarrow 0 \\
\text { as } n & \longrightarrow \infty, \\
\frac{4 M b}{(\Gamma(\mu))^{2}}\left((p-1) \frac{b^{(2 \mu p-p-1) /(p-1)}}{2 \mu p-p-1}\right)^{(p-1) / p} & \\
\cdot\left(\int_{0}^{b} E\left\|B(s) u_{n}(s)-B(s) \widehat{u}(s)\right\|^{2 p} d s\right)^{1 / p} & \longrightarrow 0 \\
\text { as } n & \longrightarrow \infty .
\end{aligned}
$$


Consequently, $E\left\|x_{n}(t)-\widehat{x}(t)\right\|^{2} \rightarrow 0$ as $n \rightarrow \infty$. Thus, by $\left(H_{4}\right)$ and Balder's theorem [40], we obtain

$$
\mathfrak{J}(\widehat{x}, \widehat{u}) \leq \lim _{n \rightarrow \infty} \mathfrak{J}\left(x_{n}, u_{n}\right)=m
$$

This shows that $\mathfrak{J}$ attains its minimum at $\widehat{u} \in U_{\text {ad }}$.

\section{Example}

In this section, we provide an example to verify the theoretical results. Consider the control problem

$$
\begin{aligned}
& D_{0^{+}}^{\nu, 3 / 4} y(t, x) \\
& =\frac{\partial^{2}}{\partial x^{2}} y(t, x)+B(t) u(t) x \\
& \quad+\frac{e^{-t} y(t, x)}{\left(1+e^{t}\right)(1+y(t, x))}+\int_{0}^{t} \frac{\sin y(t, x)}{t^{1 / 3}} d \beta(s) \\
& \quad+\int_{Z}\left(1+e^{-t}\right) \cos y(t, x) \eta \widetilde{N}(d t, d \eta), \\
& y(t, 0)=y(t, \pi)=0, \quad t>0, \quad 0 \leq x \leq \pi, u \in U_{\mathrm{ad}}, \\
& I_{0^{+}}^{(1-v)(1 / 4)} y(0)=y_{0}, \quad 0<x<\pi, \quad 0 \leq t \leq b .
\end{aligned}
$$

Here, $D_{0^{+}}^{\nu, 3 / 4}$ is the Hilfer fractional derivative, $0 \leq \nu \leq 1, \mu=$ $3 / 4, b>0$. Let $\beta(t)$ denote a standard one-dimensional Wiener process in $H=L_{2}([0, \pi])$ defined on $(\Omega, \mathfrak{F}, P)$. The operator $A: H \rightarrow H$ is defined by $A y=y^{\prime \prime}$ with the domain $D(A)=\left\{y \in H: y, y^{\prime}\right.$ absolutely continuous, $y^{\prime \prime} \epsilon$ $H, y(0)=y(\pi)=0\}$. Then $A y=\sum_{n=1}^{\infty}-n^{2}\left\langle y, y_{n}\right\rangle y_{n}$, $y \in D(A)$, where $y_{n}(x)=\sqrt{2 / \pi} \sin (n x), n \in \mathbb{N}$, is the orthogonal set of eigenvectors of $A$. It is well known that $A$ generates a compact semigroup $(T(t))_{t \geq 0}$ in $H$ and is given by $T(t) y=\sum_{n=1}^{\infty} e^{-n^{2} t}\left\langle y, y_{n}\right\rangle y_{n}$, for $y \in H$. Moreover, for any $y \in H$, we have

$$
\begin{aligned}
& \mathscr{T}_{3 / 4}(t)=\frac{3}{4} \int_{0}^{\infty} \theta \Psi_{3 / 4}(\theta) T\left(t^{3 / 4} \theta\right) d \theta \\
& \mathscr{T}_{3 / 4}(t) y \\
& \quad=\frac{3}{4} \sum_{n=1}^{\infty} \int_{0}^{\infty} \theta \Psi_{3 / 4}(\theta) \exp \left(-n^{2} t^{3 / 4} \theta\right) d \theta\left\langle y, y_{n}\right\rangle y_{n} .
\end{aligned}
$$

The Poisson point process $\{q(t) ; t \in J\}$ induced the Poisson counting measure $N(d s, d \eta)$ and the compensating martingale measure defined as

$$
\widetilde{N}(d s, d \eta)=N(d s, d \eta)-\lambda(d \eta) d s
$$

The nonlinear functions $f: J \times H \rightarrow H, g: J \times H \rightarrow L_{Q}(H)$, and $h: J \times H \rightarrow H$ are defined by

$$
\begin{aligned}
& f(y)(x)=\frac{e^{-t} y(t, x)}{\left(1+e^{t}\right)(1+y(t, x))}, \\
& g(y)(x)=\frac{\sin y(t, x)}{t^{1 / 3}} \\
& h(y)(x)=\left(1+e^{-t}\right) \cos y(t, x)
\end{aligned}
$$

and assuming that $\int_{Z} \eta^{2} \lambda(d \eta)<\infty, \int_{Z} \eta^{4} \lambda(d \eta)<\infty$. Clearly, the functions $f, g$, and $h$ satisfy the assumptions $\left(H_{1}\right)-\left(H_{2}\right)$. If $B=0$, then problem (41) can be written as the form of (1). All the conditions stated in Theorem 7 are satisfied for system (41) and can be applied to ensure the existence and uniqueness of the mild solution of (41). The controls are the functions $u: T y([0, \pi]) \rightarrow \mathbb{R}$, such that $u \in L_{2}(T y([0, \pi]))$. It means that $t \rightarrow u(\cdot, t)$ going from $J$ into $Y$ is measurable. Set $U(t)=\left\{u \in Y:\|u\|_{Y} \leq \tau_{1}\right\}$, where $\tau_{1} \in L_{2}\left(J, \mathbb{R}^{+}\right)$. We restrict the admissible controls $U_{\text {ad }}$ to be all $u \in L_{2}(T y([0, \pi]))$ such that $\|u(\cdot, t)\|^{2} \leq \tau_{1}(t)$ almost everywhere.

Let us define $B(t) u(t) x=\int_{[0, \pi]} k_{1}(x, \gamma) u(\gamma, t) d \gamma$ and make the following assumptions:

(i) $k_{1}$ is continuous.

(ii) $u \in L_{2}(J \times[0, \pi])$ and $\mathscr{L}: J \times H \times Y \rightarrow \mathbb{R} \cup\{+\infty\}$ is defined by

$$
\begin{aligned}
\mathscr{L} & \left(t, y^{u}(t), u(t)\right) \\
& =\int_{[o, \pi]}\left(\|y(t)(x)\|^{2}+\|u(t)(x)\|^{2}\right) d x .
\end{aligned}
$$

Then, system (41) can be written as in the form of (28). All the conditions stated in Theorem 10 are verified. Therefore, there exists an admissible control pair $(y, u)$ such that the associated cost functional

$$
\mathfrak{\Im}(y, u)=\int_{0}^{b} \mathscr{L}\left(t, y^{u}(t), u(t)\right) d t
$$

attains its minimum.

\section{Concluding Remarks}

In this paper, we studied the existence of solutions and optimal control results of fractional stochastic differential equations with Hilfer fractional derivative and Poisson jumps. The existence and uniqueness of mild solutions for the system have been obtained by using the successive approximation theory and stochastic analysis techniques. New sufficient conditions for optimal control results of fractional stochastic control system have been deduced. Throughout an example, the effectiveness of the obtained results is proven, under suitable conditions, for fractional stochastic partial differential equations with Poisson jumps.

The optimal control analysis for fractional stochastic differential inclusions with distributed delays, time varying delays, and impulsive effects will be our future work. 


\section{Conflicts of Interest}

The authors declare that there are no conflicts of interest regarding the publication of this paper.

\section{Acknowledgments}

This research was generously supported by the UAE University.

\section{References}

[1] A. Dzieliński, D. Sierociuk, and G. Sarwas, "Some applications of fractional order calculus," Bulletin of the Polish Academy of Sciences: Technical Sciences, vol. 58, no. 4, pp. 583-592, 2010.

[2] F. A. Rihan, "Numerical modeling of fractional-order biological systems," Abstract and Applied Analysis, vol. 2013, Article ID 816803, 2013.

[3] F. A. Rihan, S. Lakshmanan, A. H. Hashish, R. Rakkiyappan, and E. Ahmed, "Fractional-order delayed predator-prey systems with Holling type-II functional response," Nonlinear Dynamics. An International Journal of Nonlinear Dynamics and Chaos in Engineering Systems, vol. 80, no. 1-2, pp. 777-789, 2015.

[4] A. M. El-Sayed, A. E. El-Mesiry, and H. A. El-Saka, "On the fractional-order logistic equation," Applied Mathematics Letters. An International Journal of Rapid Publication, vol. 20, no. 7, pp. 817823, 2007.

[5] R. Hilfer, Applications of Fractional Calculus in Physics, World Scientific, Singapore, 2000.

[6] G. M. Zaslavsky, "Chaos, fractional kinetics, and anomalous transport," Physics Reports. A Review Section of Physics Letters, vol. 371, no. 6, pp. 461-580, 2002.

[7] S. B. Yuste, L. Acedo, and K. Lindenberg, "Reaction front in an $A+\vec{B} C$ reaction-subdiffusion process," Physical Review E: Statistical, Nonlinear, and Soft Matter Physics, vol. 69, no. 3, Article ID 036126, 2004.

[8] H. Sheng, Y. Q. Chen, and T. S. Qiu, Fractional Processes and Fractional-Order Signal Processing, Springer, New York, NY, USA, 2012.

[9] J. A. Tenreiro Machado, "Entropy analysis of integer and fractional dynamical systems," Nonlinear Dynamics. An International Journal of Nonlinear Dynamics and Chaos in Engineering Systems, vol. 62, no. 1-2, pp. 371-378, 2010.

[10] J. A. Tenreiro Machado and A. M. Galhano, "Fractional order inductive phenomena based on the skin effect," Nonlinear Dynamics. An International Journal of Nonlinear Dynamics and Chaos in Engineering Systems, vol. 68, no. 1-2, pp. 107-115, 2012.

[11] Y. Ferdi, "Some applications of fractional order calculus to design digital filters for biomedical signal processing," Journal of Mechanics in Medicine and Biology, vol. 12, no. 2, Article ID 12400088, 13 pages, 2012.

[12] W. C. Chen, "Nonlinear dynamics and chaos in a fractionalorder financial system," Chaos, Solitons and Fractals, vol. 36, no. 5, pp. 1305-1314, 2008.

[13] R. Caponetto, G. Dongola, and L. Fortuna, Fractional Order Systems: Modeling and Control Applications, World Scientific, London, UK, 2010.

[14] J. A. Tenreiro Machado, "Analysis and design of fractional-order digital control systems," Systems Analysis Modelling Simulation, vol. 27, pp. 107-122, 1997.
[15] R. Hilfer, "Experimental evidence for fractional time evolution in glass forming materials," Chemical Physics, vol. 284, no. 1-2, pp. 399-408, 2002.

[16] D. Sierociuk, A. Dzielinski, G. Sarwas, I. Petras, I. Podlubny, and T. Skovranek, "Modelling heat transfer in heterogeneous media using fractional calculus," Philosophical Transactions of the Royal Society of London. Series A. Mathematical, Physical and Engineering Sciences, vol. 371, no. 1990, Article ID 20120146, 20120146, 10 pages, 2013.

[17] A. A. Kilbas, H. M. Srivastava, and J. J. Trujillo, "Preface," NorthHolland Mathematics Studies, vol. 204, no. C, pp. vii-x, 2006.

[18] T. Sandev, R. Metzler, and Z. Tomovski, "Fractional diffusion equation with a generalized Riemann-Liouville time fractional derivative," Journal of Physics. A. Mathematical and Theoretical, vol. 44, no. 25, Article ID 255203, 255203, 21 pages, 2011.

[19] N. I. Mahmudov and M. A. McKibben, "On the approximate controllability of fractional evolution equations with generalized Riemann-Liouville fractional derivative," Journal of Function Spaces, Article ID 263823, Art. ID 263823, 9 pages, 2015.

[20] H. Gu and J. J. Trujillo, "Existence of mild solution for evolution equation with Hilfer fractional derivative," Applied Mathematics and Computation, vol. 257, pp. 344-354, 2015.

[21] K. M. Furati, M. D. Kassim, and N. e. Tatar, "Existence and uniqueness for a problem involving Hilfer fractional derivative," Computers \& Mathematics with Applications. An International Journal, vol. 64, no. 6, pp. 1616-1626, 2012.

[22] V. Lakshmikantham, S. Leela, and J. V. Devi, Theory of Fractional Dynamic Systems, Cambridge Scientific Publishers, Cambridge, UK, 2009.

[23] E. Gerolymatou, I. Vardoulakis, and R. Hilfer, "Modelling infiltration by means of a nonlinear fractional diffusion model," Journal of Physics D: Applied Physics, vol. 39, no. 18, article no. 022, pp. 4104-4110, 2006.

[24] R. Hilfer, Y. Luchko, and Z. Tomovski, "Operational method for the solution of the fractional differential equations with generalized Riemann-Liouville fractional derivatives," Fractional Calculus and Applied Analysis, vol. 12, pp. 299-318, 2009.

[25] P. Chen and Y. Li, "Nonlocal Cauchy problem for fractional stochastic evolution equations in Hilbert spaces," Collectanea Mathematica, vol. 66, no. 1, pp. 63-76, 2015.

[26] J. Wang, "Approximate mild solutions of fractional stochastic evolution equations in Hilbert spaces," Applied Mathematics and Computation, vol. 256, pp. 315-323, 2015.

[27] L. Lu and Z. Liu, "Existence and controllability results for stochastic fractional evolution hemivariational inequalities," Applied Mathematics and Computation, vol. 268, pp. 1164-1176, 2015.

[28] R. C. Merton, "Option pricing when underlying stock returns are discontinuous," Journal of Financial Economics, vol. 3, no. 1-2, pp. 125-144, 1976.

[29] P. Jorion, "On jump processes in the foreign exchange and stock markets," Review of Financial Studies, vol. 1, pp. 427-445, 1988.

[30] Y. Ren, Q. Zhou, and L. Chen, "Existence, uniqueness and stability of mild solutions for time-dependent stochastic evolution equations with Poisson jumps and infinite delay," Journal of Optimization Theory and Applications, vol. 149, no. 2, pp. 315331, 2011.

[31] C. Rajivganthi and P. Muthukumar, "Almost automorphic solutions for fractional stochastic differential equations and its optimal control," Optimal Control Applications \& Methods, vol. 37, no. 4, pp. 663-681, 2016. 
[32] S. Hosseinpour and A. Nazemi, "Solving fractional optimal control problems with fixed or free final states by Haar wavelet collocation method," IMA Journal of Mathematical Control and Information, vol. 33, no. 2, pp. 543-561, 2016.

[33] R. Kamocki and M. Majewski, "Fractional linear control systems with Caputo derivative and their optimization," Optimal Control Applications \& Methods, vol. 36, no. 6, pp. 953-967, 2015.

[34] Z. Fan and G. Mophou, "Existence of optimal controls for a semilinear composite fractional relaxation equation," Reports on Mathematical Physics, vol. 73, no. 3, pp. 311-323, 2014.

[35] J. Wang, Y. Zhou, and M. Medved, "On the solvability and optimal controls of fractional integrodifferential evolution systems with infinite delay," Journal of Optimization Theory and Applications, vol. 152, no. 1, pp. 31-50, 2012.

[36] J. Ren and J. Wu, "The optimal control problem associated with multi-valued stochastic differential equations with jumps," Nonlinear Analysis. Theory, Methods \& Applications. An International Multidisciplinary Journal, vol. 86, pp. 30-51, 2013.

[37] N. U. Ahmed, "Stochastic initial boundary value problems subject to distributed and boundary noise and their optimal control," Journal of Mathematical Analysis and Applications, vol. 421, no. 1, pp. 157-179, 2015.

[38] C. Rajivganthi, P. Muthukumar, and B. Ganesh Priya, "Successive approximation and optimal controls on fractional neutral stochastic differential equations with Poisson jumps," Optimal Control Applications \& Methods, vol. 37, no. 4, pp. 627-640, 2016.

[39] Y. Ren, X. Cheng, and R. Sakthivel, "Impulsive neutral stochastic functional integro-differential equations with infinite delay driven by fBm," Applied Mathematics and Computation, vol. 247, pp. 205-212, 2014.

[40] E. J. Balder, "Necessary and sufficient conditions for $L_{1}$-strongweak lower semicontinuity of integral functionals," Nonlinear Analysis. Theory, Methods \& Applications. An International Multidisciplinary Journal, vol. 11, no. 12, pp. 1399-1404, 1987. 


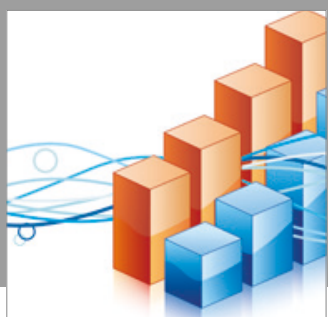

Advances in

Operations Research

vatersals

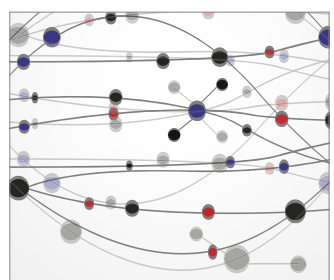

\section{The Scientific} World Journal
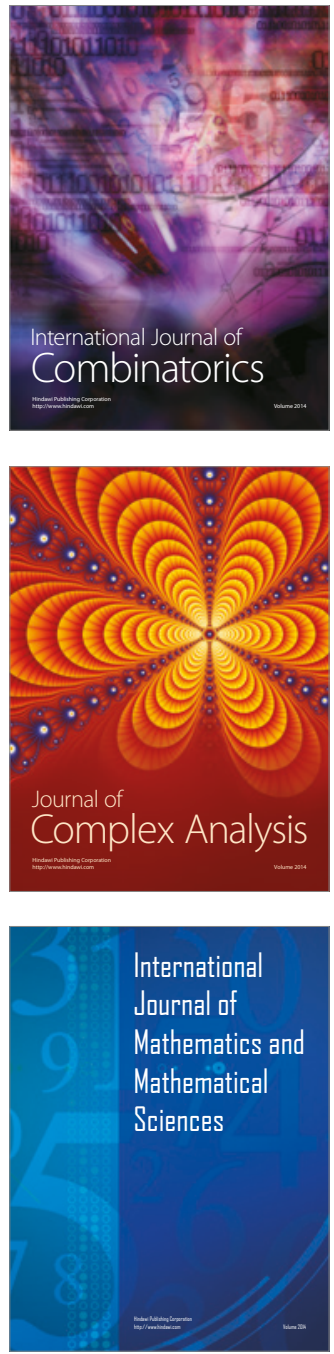
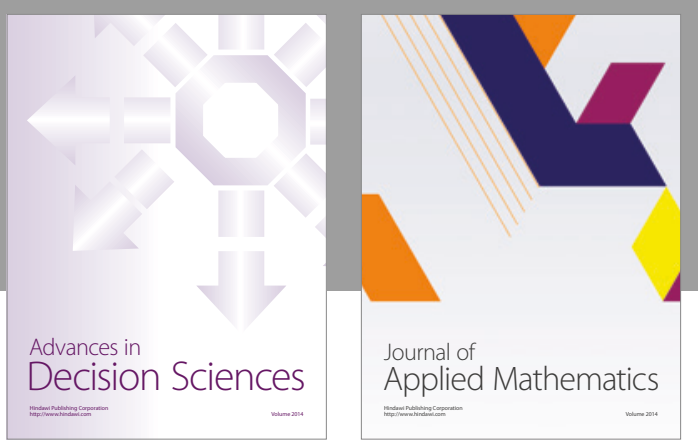

Algebra

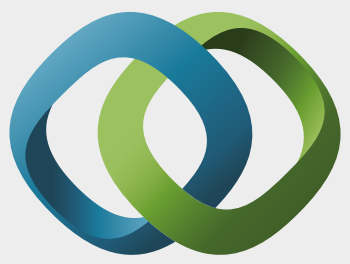

\section{Hindawi}

Submit your manuscripts at

https://www.hindawi.com
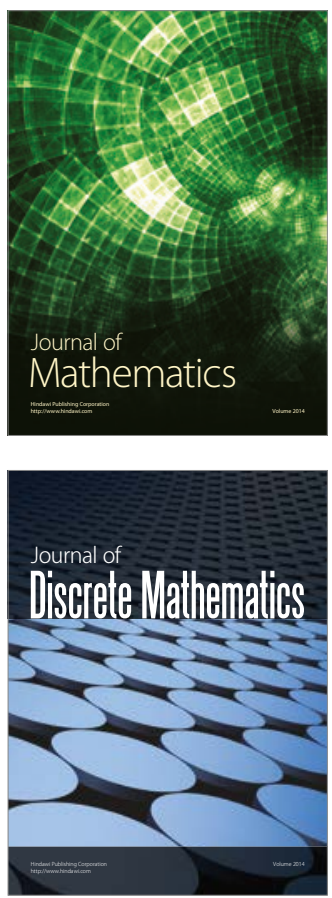

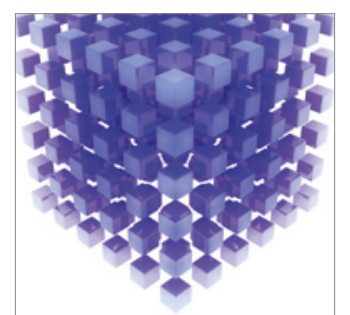

Mathematical Problems in Engineering
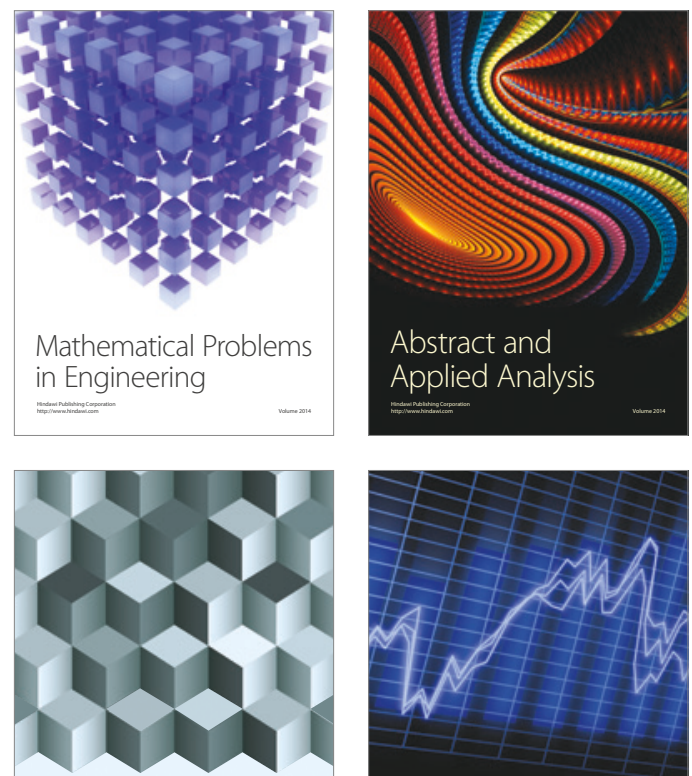

Journal of

Function Spaces

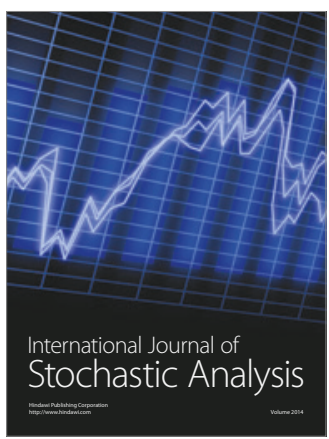

Probability and Statistics
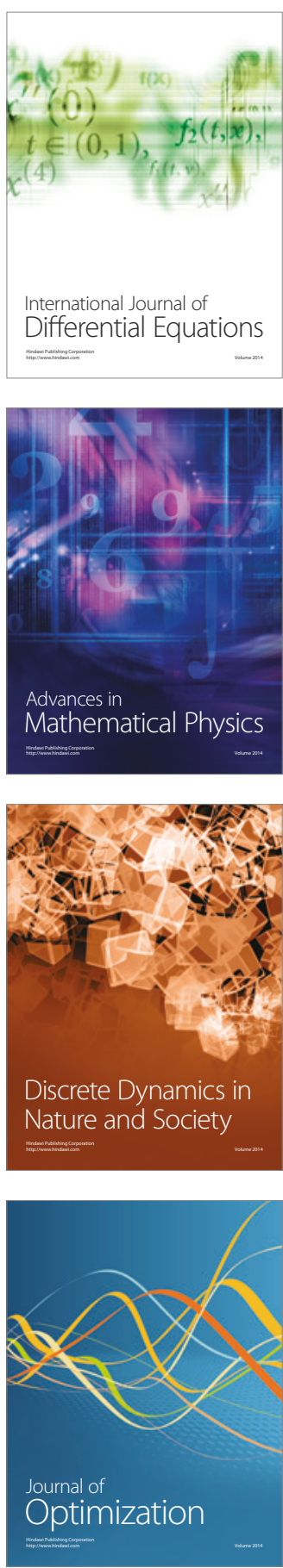